\title{
Diarrea crónica como manifestación de un tumor neuroendocrino
}

\section{Chronic diarrhea as a manifestation of a neuroendocrine tumor}

Héctor Fabio Sandoval A., MD, ${ }^{*}$ Angélica María González C., MD, ${ }^{2}$ María Camila Angarita C., MD, ${ }^{3}$ Micela Beatriz Rosado R., MD, ${ }^{4}$

Rubén Darío Arenas D., MD. ${ }^{5}$

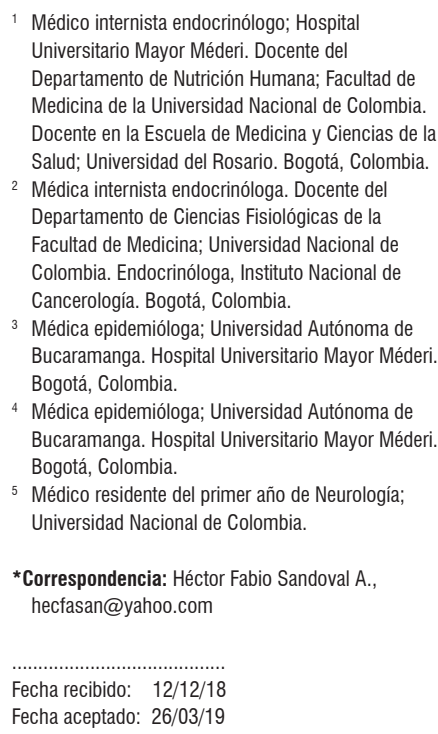

Médico internista endocrinólogo; Hospital Universitario Mayor Méderi. Docente del

Departamento de Nutrición Humana; Facultad de Medicina de la Universidad Nacional de Colombia. Docente en la Escuela de Medicina y Ciencias de la Salud; Universidad del Rosario. Bogotá, Colombia.

2 Médica internista endocrinóloga. Docente del Departamento de Ciencias Fisiológicas de la Facultad de Medicina; Universidad Nacional de Colombia. Endocrinóloga, Instituto Nacional de Cancerología. Bogotá, Colombia.

3 Médica epidemióloga; Universidad Autónoma de Bucaramanga. Hospital Universitario Mayor Méderi. Bogotá, Colombia.

${ }^{4}$ Médica epidemióloga; Universidad Autónoma de Bucaramanga. Hospital Universitario Mayor Méderi. Bogotá, Colombia.

5 Médico residente del primer año de Neurología; Universidad Nacional de Colombia.

*Correspondencia: Héctor Fabio Sandoval A., hecfasan@yahoo.com

Fecha recibido: $\quad 12 / 12 / 18$

Fecha aceptado: 26/03/19

\begin{abstract}
Resumen
Las neoplasias neuroendocrinas (Neuroendocrine Neoplasms, NEN) constituyen un grupo heterogéneo de neoplasias poco frecuentes, que se originan en las células endocrinas, con la capacidad de secretar aminas y polipéptidos hormonales. Las NEN de localización pancreática ( $p N E N$ ) pueden ser funcionales o no funcionales. Las pNEN funcionales secretan hormonas como la gastrina, la insulina y el glucagón y otras menos frecuentes como el péptido intestinal vasoactivo (PIV), por lo que sus características sindromáticas dependen del péptido secretado. Los vipomas se manifiestan con diarrea crónica de características secretoras, que usualmente conducen a trastornos hidroelectrolíticos e incluso a complicaciones serias asociadas como la falla renal. A continuación, se describe el caso de un hombre de 37 años con diarrea crónica de 6 meses de evolución y frecuentes hospitalizaciones por trastornos hidroelectrolíticos, generados por hipocalemia severa y lesión renal aguda por deshidratación. Después de múltiples estudios, se considera el diagnóstico de una diarrea secretora por NEN funcional, secretora de PIV. Por tanto, se inicia una terapia empírica con octreotida y se logra controlar la diarrea, así como corregir el trastorno hidroelectrolítico. Además, se amplían los estudios, para documentar las pNEN tratadas mediante intervención quirúrgica, con respuesta clínica favorable y remisión completa de la sintomatología.
\end{abstract}

\section{Palabras clave}

Diarrea crónica, diarrea secretora, tumor neuroendocrino, vipoma.

\section{Abstract}

Neuroendocrine tumors (NETs) are a heterogeneous group of rare neoplasms that originate in endocrine cells with the ability to secrete amines and hormonal polypeptides. Pancreatic neuroendocrine tumors (PNETs) can be functional or non-functional. Functional PNETs secrete common hormones such as gastrin, insulin and glucagon and much less frequent hormones such as vasoactive intestinal peptide (VIP). Their characteristics depend on the peptide secreted. Vipomas are characterized by chronic diarrhea of secretory characteristics that usually lead to hydroelectrolytic disorders and can lead to serious complications associated with renal failure. This article describes the case of a 37-year-old man who had suffered chronic diarrhea with frequent hospitalization for hydroelectrolytic disorders for six months due to severe hypokalemia and acute renal damage due to dehydration. After multiple studies, a diagnosis of secretory diarrhea due to a VIP secretory functional NET was considered. Empirical therapy with Octreotide was begun to control diarrhea and correct the hydroelectrolytic disorder. More studies of PNETS are being published. They have been treated surgically intervention with favorable clinical results and complete remission of symptoms.

\section{Keywords}

Chronic diarrhea, secretory diarrhea, neuroendocrine tumor, vipoma. 


\section{INTRODUCCIÓN}

La diarrea se define como una reducción significativa en la consistencia de las deposiciones o un aumento en el volumen de estas, $>200 \mathrm{~g} / \mathrm{d}$. Generalmente, se asocia a molestias abdominales o una urgencia por defecar. De acuerdo con el tiempo de evolución, se considera diarrea crónica a aquella que tiene una duración $>3$ semanas, y algunos autores la describen como $>4$ semanas (1-3).

Respecto a su clasificación, se divide en tres grandes grupos: 1) diarrea acuosa, que a su vez se subdivide en osmótica -en la que ocurre una retención de agua intraluminal por sustancias no absorbidas-, secretora -en la cual la absorción de agua es reducida- $y$ funcional, generada por hipermotilidad; 2) diarrea grasa (esteatorrea), que se subdivide en los síndromes de malabsorción -en el que ocurre un daño o la pérdida de la capacidad de absorción-y de maldigestión, ocasionado por una pérdida de la función digestiva, y 3 ) diarrea inflamatoria o exudativa, la cual se presenta con leucocitosis y la existencia de leucocitos en la materia fecal, e incluso con la presencia de material purulento y sangre franca (4).

La diarrea secretora, incluida dentro de la diarrea acuosa, se origina por un aumento de la secreción de electrolitos (especialmente sodio y cloro) hacia el lumen intestinal, lo cual genera un arrastre de agua, por causa de una alteración del epitelio intestinal en el transporte de agua y de electrolitos (2).

Clínicamente, la diarrea secretora se caracteriza por no estar en relación con la ingesta, por lo que con frecuencia los pacientes describen su aparición como de predominio nocturno. Por tanto, tampoco existe una respuesta al ayuno. Así, el volumen fecal no se disminuye ni se aumenta en caso de ingesta (4) y su osmolaridad es similar a la del plasma (fecal osmotic gap $<50 \mathrm{mOsm} / \mathrm{kg}$ ) (4). Además, la diarrea secretora es voluminosa, incluso con un gasto $>1 \mathrm{~L}$ en $24 \mathrm{~h}$, y se relaciona habitualmente con trastornos electrolíticos como hipocalemia y acidosis metabólica por la pérdida concomitante de bicarbonato (4).

Las causas de la diarrea secretora son múltiples y entre ellas se incluyen etiologías infecciosas, como las generadas por las enterotoxinas del Vibrio cholerae y de la Escherichia coli. Asimismo, se suman las causas posquirúrgicas (gastrectomía, resección intestinal, vagotomía); los secretagogos luminales (como los ácidos biliares o laxantes); los secretagogos circulantes, como algunos medicamentos (digitálicos, biguanidas, misoprostol), las hormonas (exceso de hormona tiroidea) $(5,6)$, y las NEN funcionales, productoras de péptidos como la gastrina, el glucagón y, menos frecuentemente, el PIV, como lo es el caso clínico descrito en este reporte.

Dentro de este contexto, las NEN son neoplasias que se presentan con una frecuencia relativamente baja, de pre- sentación clínica muy variable y que pueden pasar inicialmente desapercibidas. Sin embargo, poseen un potencial maligno y metastásico.

En efecto, el $85 \%$ de las NEN se originan en el tracto gastrointestinal, mientras que un $10 \%$ ocurren en el pulmón y se manifiestan como carcinoides bronquiales (7). Entre tanto, las pNEN son, en su mayoría, no funcionales. No obstante, algunas de ellas tienen la capacidad de secretar hormonas que derivan en síndromes clínicos únicos como los vipomas.

\section{CASO CLíNICO}

Se expone el caso de un paciente de 37 años, quien consultó al servicio de urgencias por un cuadro clínico, de 6 meses de evolución, con deposiciones diarreicas, abundantes, sin moco ni sangre y una frecuencia de 4 a 6 evacuaciones por día. Estas persistieron a pesar del ayuno. El paciente negó la existencia de antecedentes de importancia.

Asimismo, el paciente consultó en varias oportunidades al servicio de urgencias, por causa de síntomas asociados a deshidratación y trastornos electrolíticos con hipocalemia severa y acidosis metabólica. Por tanto, requirió en múltiples ocasiones de hospitalización. Durante el ingreso a nuestra institución, el individuo persistió con diarrea de alto gasto, así como con deshidratación moderada y trastorno electrolítico (Tabla $\mathbf{1}$ ).

El abordaje diagnóstico inicial incluyó la búsqueda de causas infecciosas, pero tanto los coprocultivos como los hemocultivos fueron negativos. Pese a que recibió múltiples esquemas antibióticos empíricos previos, no se evidenció una mejoría. La infección por VIH también fue descartada. Fue valorado por el servicio de gastroenterología, en donde se le practicaron una endoscopia de vías digestivas altas (EVDA) y una colonoscopia avanzada a íleon con toma de biopsias. Estas pruebas descartaron la presencia de una enfermedad inflamatoria intestinal y de una enfermedad celíaca. Esta última también fue excluida por el reporte negativo para anticuerpos antiendomisio.

La diarrea se catalogó como secretora (voluminosa, no relacionada con la ingesta alimentaria) persistiendo y haciéndose más intensa aún, conduciendo a choque hipovolémico que requirió soporte vasopresor, hipocalemia severa persistente que necesitó reposición con potasio parenteral e incluso, provocó una lesión renal aguda con necesidad de iniciar terapia de reemplazo renal. En este escenario, se sospecha de una NEN funcional y se solicita el apoyo de endocrinología.

Dadas las características descritas de la diarrea, así como la existencia de la hipocalemia severa, la acidosis metabólica con depleción severa en los niveles de bicarbonato y la presencia de hipercalcemia, se decide solicitar niveles de 
Tabla 1. Principales estudios realizados durante la hospitalización, previo (reporte) y posterior (control) al uso de octreotida.

\begin{tabular}{|c|c|c|}
\hline Parámetro & Reporte & Control \\
\hline Potasio & $1,3 \mathrm{mEq} / \mathrm{L}$ & $3,6 \mathrm{mEq} / \mathrm{L}$ \\
\hline Cloro & $123 \mathrm{mEq} / \mathrm{L}$ & $96 \mathrm{mEq} / \mathrm{L}$ \\
\hline Sodio & $132 \mathrm{mEq} / \mathrm{L}$ & $140 \mathrm{mEq} / \mathrm{L}$ \\
\hline Calcio ionizado & $1,92 \mathrm{mEq} / \mathrm{L}$ & $1,25 \mathrm{mEq} / \mathrm{L}$ \\
\hline Gases arteriales & $\begin{array}{c}\mathrm{pH}: 7,22 ; \mathrm{pO}_{2}: 90 ; \mathrm{pCO}_{2}: \\
18 ; \mathrm{HCO}_{3}: \\
7 \mathrm{~EB}:-18,3\end{array}$ & $\begin{array}{l}\mathrm{pH}: 7,4 ; \mathrm{pO}_{2}: 71 ; \\
\mathrm{pCO}_{2}: 33 ; \mathrm{HCO}_{3}: \\
21 \mathrm{~EB}:-2\end{array}$ \\
\hline Creatinina & $6,73 \mathrm{mg} / \mathrm{dL}$ & $1,23 \mathrm{mg} / \mathrm{dL}$ \\
\hline Nitrógeno ureico & $73 \mathrm{mg} / \mathrm{mL}$ & $15,9 \mathrm{mg} / \mathrm{mL}$ \\
\hline Potasio en orina & $7,26 \mathrm{mEq} / \mathrm{L}$ & - \\
\hline TSH & $3,1 \mathrm{mU} / \mathrm{L}$ & - \\
\hline PTH & 4,5 & - \\
\hline $\begin{array}{l}\text { Ácido indolacético en } \\
\text { orina } 24 \text { h (5-HIAA) }\end{array}$ & Negativo & - \\
\hline Cromogranina $\mathrm{A}(\mathrm{CgA})$ & $57,4 \mathrm{ng} / \mathrm{mL}$ & \\
\hline Albúmina & $4,1 \mathrm{~g} / \mathrm{dL}$ & - \\
\hline $\begin{array}{l}\text { Anticuerpo } \lg A \\
\text { antiendomisio }\end{array}$ & No reactivo & - \\
\hline $\begin{array}{l}\text { Biopsia renal } \\
\text { extrainstitucional }\end{array}$ & $\begin{array}{l}\text { Necrosis tubular aguda, } \\
\text { cambios histológicos } \\
\text { compatibles con } \\
\text { nefropatía asociada a } \\
\text { hipocalemia. Puntuación } \\
\text { total de cronicidad = } 0\end{array}$ & - \\
\hline
\end{tabular}

$\mathrm{mEq}$ : miliequivalente; $\mathrm{pO}_{2}$ : presión parcial de oxígeno; $\mathrm{pCO}_{2}$ : presión parcial de dióxido de carbono; $\mathrm{HCO}_{3}$ : bicarbonato; $\mathrm{EB}$ : exceso de base; 5-HIAA: ácido 5-hidroxindolacético; TSH: hormona estimulante de la tiroides (Thyroid-Stimulating Hormone); PTH: hormona paratiroidea; IgA: inmunoglobulina A
PIV. Sin embargo, ante la no disponibilidad de realizar la medición en nuestro medio, y dada la gravedad del cuadro, se realiza una prueba terapéutica con octreotida de $0,1 \mu \mathrm{g}$ subcutáneos, cada $8 \mathrm{~h}$.

La terapia con el análogo de somatostatina condujo a la remisión total de la diarrea en las siguientes 48 a $72 \mathrm{~h}$ y a la resolución del choque y de la lesión renal aguda, con la suspensión, incluso, de la terapia de reemplazo renal.

Para confirmar la impresión diagnóstica de vipoma, que ganó fuerza con la respuesta positiva a la prueba terapéutica con octreotida, se realizó una resonancia magnética nuclear (RMN) de abdomen con contraste, la cual mostró una masa sólida hipervascular de $22 \mathrm{~mm}$ en la cola del páncreas (Figura 1), sin lesiones metastásicas. La lesión era susceptible de manejo quirúrgico, por lo que el paciente fue dirigido a una pancreatectomía distal laparoscópica más esplenectomía, por el grupo de cirugía hepatobiliar.

El estudio histopatológico demostró la presencia de una NEN de páncreas grado 2 (OMS), un tamaño tumoral de $2,2 \times 2 \mathrm{~cm}$, un Ki67 del $5 \%$, con conteo mitótico de 2 mitosis por 10 campos de alto poder. No se identificó una invasión linfovascular o perineural. Durante el posoperatorio, se suspendió la octreotida, a fin de determinar el éxito del procedimiento quirúrgico, sin que sucediera la reaparición de las deposiciones diarreicas. En la actualidad, el paciente se encuentra libre de síntomas.

\section{DISCUSIÓN}

Las pNEN son un grupo no muy frecuente de neoplasias que surgen de células madre multipotentes en el epitelio ductal pancreático (8). El $50 \%$ de estas son no funcionales
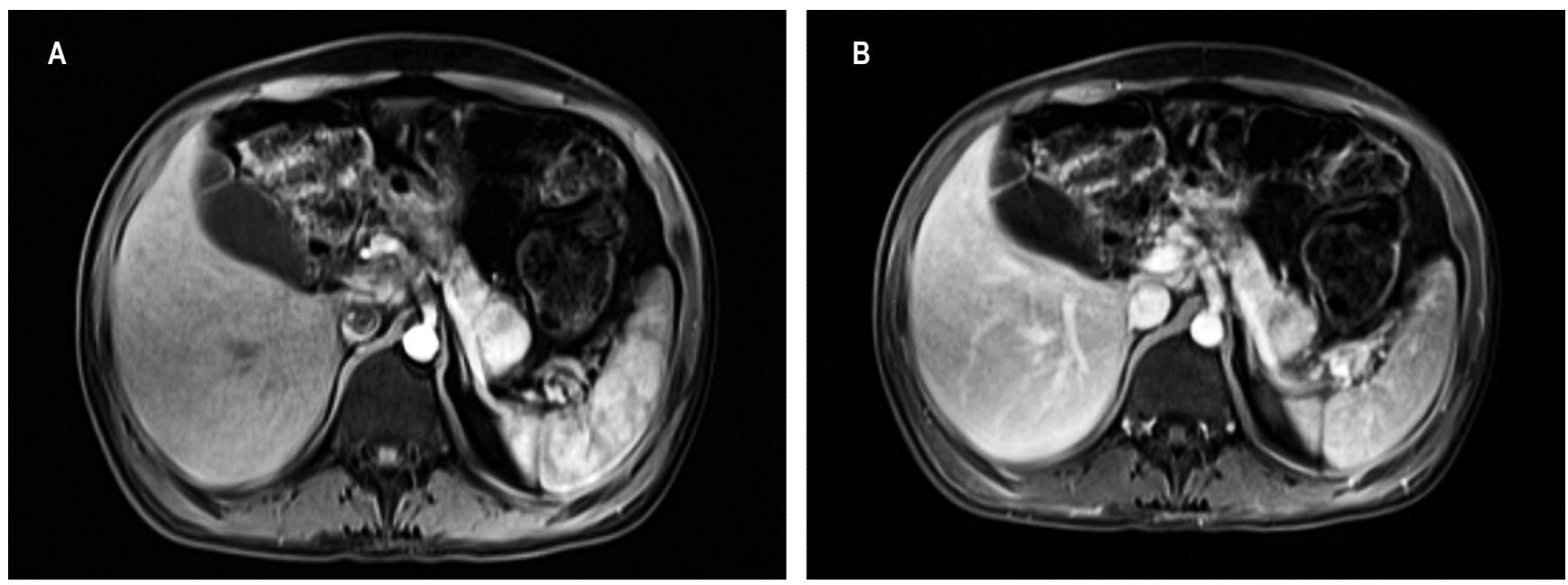

Figura 1. A. RMN abdominal contrastada. Lesión sólida de $22 \mathrm{~mm}$ en la cola del páncreas, con realce posterior; administración de contraste endovenoso. Páncreas de tamaño normal. B. Lesión sólida de $22 \mathrm{~mm}$ en la cola del páncreas, hipointensa en T1, isointensa en T2, hiperintensa en difusión, con realce posterior a la administración de contraste endovenoso. 
o secretan péptidos de bajo poder biológico, como el polipéptido pancreático (PP) o la neurotensina. El otro $50 \%$ son funcionales y los productores de gastrina (gastrinoma) e insulina (insulinoma) son las más comunes.

Los insulinomas, caracterizados clínicamente por producir hipoglucemia con síntomas neuroglicopénicos, son benignos hasta en el $90 \%$ de los casos (7). Entre tanto, los gastrinomas se caracterizan por la presencia de úlceras pépticas, diarreas severas y reflujo gastroesofágico secundario a una elevada producción de gastrina $(7,8)$. Los glucagonomas se manifiestan por diabetes mellitus, trombosis, depresión y una erupción cutánea conocida como eritema necrolítico migratorio, el cual se presenta principalmente en los miembros inferiores, como una lesión eritematosa de color rojo marrón, exfoliativa y con necrólisis superficial $(5,6)$.

De otro lado, los vipomas (o síndrome de VernerMorrison) fueron descritos por primera vez en $1958(7,8)$, constituyendo una enfermedad muy rara, con una incidencia de 1 caso por cada 10000 personas por año. Estos ocurren en los islotes B pancreáticos no productores de insulina, usualmente de gran tamaño (el $72 \%,>5 \mathrm{~cm}$ ) y malignos (64$92 \%)$, al momento del diagnóstico $(7,9)$. Generan diarrea profusa, hipocalemia y aclorhidria clásica por la secreción de PIV en el plasma, así como un polipéptido de 28 aminoácidos ampliamente distribuido en el tracto gastrointestinal y el cerebro, el cual ejerce una acción relajante sobre el músculo liso vascular y visceral. Todo ello conduce a la secreción de líquidos y electrolitos en el lumen intestinal $(6,10,11)$.

La diarrea secretora, a diferencia de la diarrea osmótica, persiste durante el ayuno. Se produce por una mayor secreción de agua y electrolitos hacia la luz intestinal, que supera la capacidad de absorción (6) y es secundaria a factores externos como el PIV, que estimulan la producción de monofosfato de adenosina (Adenosine Monophosphate, AMP) cíclico (12).

Cuando el PIV se segrega en grandes cantidades en las NEN, los pacientes suelen experimentar diarrea severa, deshidratación, eritema y pérdida de peso. El PIV también promueve la glucogenólisis hepática que se traduce en hiperglucemia, dilata los vasos sanguíneos sistémicos periféricos e inhibe la secreción de ácido clorhídrico (13).

En el abordaje diagnóstico, los estudios imagenológicos desempeñan un papel importante, ya que permiten determinar la extensión de la lesión, el compromiso de los órganos vecinos, la infiltración y la relación con estructuras vasculares y nerviosas. En primera instancia, la ecografía transabdominal ofrece una sensibilidad que varía entre un 20 y un $86 \%$ según el tamaño tumoral. Sin embargo, supone dificultades para visualizar los tumores de la superficie del páncreas y del duodeno (14). En consecuencia, este examen es útil como guía para la biopsia percutánea, en caso de estar indicada.
En contraste, la ultrasonografía endoscópica (USE) es muy útil en casos de tumores situados en la cabeza del páncreas y en la pared duodenal (14). No obstante, al considerarse el potencial de malignidad de los pNEN (15), no permite evaluar las lesiones extrapancreáticas.

Por otro lado, la tomografía computarizada (TC) permite la localización del tumor primario y de posibles lesiones secundarias, con una sensibilidad del $30 \%$ para tumores de $1 \mathrm{a} 3 \mathrm{~cm}$, y un $95 \%$ para los tumores $>3 \mathrm{~cm}(14,16)$, con una notable mejoría de su rendimiento mediante el uso de técnicas multifásicas.

En el caso de lesiones pequeñas, la RMN con gadolinio cuenta con la mayor sensibilidad (91 al 94\%) (14); sin embargo, tanto la TC multifásica como la RMN permiten la planeación del abordaje quirúrgico y su seguimiento posterior $(14,17)$.

Respecto al tratamiento, en primera instancia es fundamental corregir el trastorno hidroelectrolítico potencialmente fatal y, una vez definido el compromiso por vía imagenológica, proponer al paciente la resección quirúrgica. Si el tumor no se puede eliminar por completo, la reducción quirúrgica puede tener un beneficio paliativo asociado a terapias como los análogos de somatostatina -los cuales proveen un efecto sintomático antisecretor y antiproliferativo-, a los inhibidores de la tirosina-cinasa, como el sunitinib, o a los inhibidores de mTOR, como el everolimus (11).

En síntesis, el abordaje diagnóstico del paciente descrito en este caso clínico estuvo limitado parcialmente, al no haberse podido procesar los niveles séricos de PIV. Sin embargo, se obtuvo una confirmación diagnóstica desde el punto de vista histopatológico de una pNEN.

Tras exponer el caso del paciente del Hospital Universitario Mayor Méderi y la Clínica Fundadores, se pretende socializar y demostrar nuestra experiencia con dicha patología, para lo cual partimos de un motivo de consulta relativamente frecuente: la diarrea crónica. Se presentaron dificultades para realizar un enfoque inicial, pero, finalmente, el paciente fue diagnosticado y tratado de forma correcta hasta su resolución.

\section{CONCLUSIONES}

El vipoma es una enfermedad muy rara, con una incidencia de 1 caso por cada 10000 personas al año. Se manifiesta a partir de una diarrea secretora profusa, como síntoma principal, el cual frecuentemente conduce a hipocalemia, pérdida de peso y aclorhidria. Esta patología puede o no ser de comportamiento maligno. Sin embargo, independiente de ello, es de vital importancia reconocer esta entidad como potencialmente fatal, dadas las alteraciones hidroelectrolíticas severas que genera.

La resección quirúrgica sigue siendo el pilar de tratamiento y se ha observado la remisión completa de la sin- 
tomatología, tal y como ocurrió en el caso clínico antes expuesto. El objetivo final de este tipo de documentos es incentivar a la publicación de casos de características similares, para así unificar métodos de abordaje de diagnóstico temprano que permitan minimizar complicaciones.

\section{Conflicto de intereses}

Los autores no declaran ningún conflicto de interés.

\section{REFERENCIAS}

1. Fernández F, Esteve E. Diarrea crónica. En: Montoro MA, García JC. Gastroenterología y hepatología: problemas comunes en la práctica clínica. Barcelona: Jarpyo Editores, 2a edición; 2012. p. 125-146.

2. Pineda LF, Otero W, Arbeláez V. Diarrea crónica. Diagnóstico y evaluación clínica. Rev Col Gastroenterol. 2004;19(2):115-126.

3. Higuera-de la Tijera MF, Alexanderson-Rosas EG, ServínCaamaño AI. Abordaje del paciente con diarrea crónica. Med Int Mex. 2010;26(6):583-589.

4. Juckett G, Trivedi R. Evaluation of chronic diarrhea. Am Fam Physician. 2011 15;84(10):1119-26.

5. Schiller LR. Secretory diarrhea. Curr Gastroenterol Rep. 1999;1(5):389-97. https://doi.org/10.1007/s11894999-0020-8

6. Thiagarajah JR, Donowitz M, Verkman AS. Secretory diarrhoea: mechanisms and emerging therapies. Nat Rev Gastroenterol Hepatol. 2015;12(8):446-57. https://doi. org/10.1038/nrgastro.2015.111

7. Vargas CC, Castaño R. Tumores neuroendocrinos gastroenteropancreáticos. Rev Col Gastroenterol. 2010;25(2):165176.

8. Sívori E, Blanco D. Tumores neuroendocrinos del páncreas. Cirugía Digest. 2009;IV-489:1-9.
9. Dasari A, Shen C, Halperin D, Zhao B, Zhou S, Xu Y, et al. Trends in the Incidence, Prevalence, and Survival Outcomes in Patients With Neuroendocrine Tumors in the United States. JAMA Oncol. 2017;3(10):1335-1342. https://doi. org/10.1001/jamaoncol.2017.0589

10. André R, Koessler T, Polet D, Roth A, Ritz M, Kherad O. VIPoma : a rare etiology of diarrhea with hypokalemia. Rev Med Suisse. 2018;14(592):289-293.

11. Cavalli T, Giudici F, Santi R, Nesi G, Brandi ML, Tonelli F. Ventricularfibrillation resulting from electrolyteimbalance reveals vipoma in MEN1 syndrome. Fam Cancer. 2016;15(4):645-9. https://doi.org/10.1007/s10689-016-9906-4

12. Belei OA, Heredea ER, Boeriu E, Marcovici TM, Cerbu S, Mărginean $\mathrm{O}$, et al. Verner-Morrison syndrome. Literature review. Rom J Morphol Embryol. 2017;58(2):371-376.

13. Dimitriadis GK, Weickert MO, Randeva HS, Kaltsas G, Grossman A. Medical management of secretory syndromes related to gastroenteropancreaticneuroendocrine tumours. Endocr Relat Cancer. 2016;23(9):R423-36. https://doi. org/10.1530/ERC-16-0200

14. Díez JJ. Iglesias P. Pruebas de imagen en el diagnóstico de los tumores neuroendocrinos. Med Clin (Barc). 2010;135(7):319-325. https://doi.org/10.1016/j.medcli.2009.04.048

15. Anderson CW, Bennett JJ. Clinical Presentation and Diagnosis of Pancreatic Neuroendocrine Tumors. Surg Oncol Clin N Am. 2016;25(2):363-74. https://doi. org/10.1016/j.soc.2015.12.003.

16. Varas-Lorenzo MJ, Cugat E, Capdevila J, Sánchez-Vizcaíno E. Detección de tumores neuroendocrinos pancreáticos: 23 años de experiencia. Rev Gastroenterol Mex.2019;84(1):1825. https://doi.org/10.1016/j.rgmx.2018.02.015

17. Muros de Fuentes MA. Técnicas de imagen en el diagnóstico de los tumores neuroendocrinos gastroenteropancreáticos. Cir Andal. 2009;20:19-24. 